\title{
Bow Hunter's Stroke Due to Prominent Degenerative Spinal Disorder
}

\author{
L. Andereggen • M. Arnold $\cdot$ R. H. Andres $\cdot$ A. Raabe $\cdot$ \\ M. Reinert · J. Gralla
}

Received: 18 February 2012 / Accepted: 7 May 2012 / Published online: 12 June 2012

(C) Springer-Verlag 2012

\section{Introduction}

Symptomatic vertebrobasilar insufficiency resulting from neck rotation, also named bow hunter's syndrome due to the rotated position of the patient's head or neck when symptoms develop, most commonly occurs from temporary stenosis or occlusion of the vertebral artery (VA) at the $\mathrm{C} 1$ or $\mathrm{C} 2$ level [1-4]. There are few cases of bow hunter's syndrome caused by obstruction of the VA at the lower cervical levels between C3-C6 [5-7]. This report illustrates the case of bow hunter's syndrome at the level C5-C6 due to a degenerative osseous prominence in a woman presenting with acute ischemic stroke finally treated by surgical decompression.

\section{Case Report}

A 66-year old previously healthy female patient was admitted to our Stroke Unit due to sudden nondirectional vertigo, vomiting, and temporary loss of consciousness while looking

\section{J. Gralla, M.D. M.Sc. $(\bowtie)$}

Institute of Diagnostic and Interventional Neuroradiology,

Bern University Hospital, 3010 Bern, Switzerland

e-mail: jan.gralla@insel.ch

\section{Andereggen, M.D.}

Department of Neurosurgery and Institute of Diagnostic and Interventional Neuroradiology

L. Andereggen, M.D. · R. H. Andres, M.D. ·

A. Raabe, M.D., Ph.D. · M. Reinert, M.D.

Department of Neurosurgery, Bern University Hospital,

Bern, Switzerland

M. Arnold, M.D.

Department of Neurology, Bern University Hospital,

Bern, Switzerland out of a train window. Clinical examination revealed horizontal nystagmus and a positive Babinski sign on the left side. Routine serum lab values were normal, and the echocardiogram was unremarkable. Magnetic resonance imaging (MRI) revealed the presence of an acute stroke in the left cerebellar hemisphere corresponding to the posterior inferior cerebellar artery (PICA) territory (Fig. 1a and b). Magnetic resonance angiography (MRA) showed severe stenosis due to thrombus formation in the V2 segment of the VA on the left side in addition to contralateral VA hypoplasia. No stenosis of the other supraaortal vessels was detected. Intravenous thrombolysis was initiated using a recombinant tissue plasminogen activator and subsequently, we performed digital subtraction angiography (DSA) for possible endovascular intervention (Fig. 1c). There was a partial recanalization of the left VA; therefore, no endovascular recanalization was performed.

Within a few hours, the patient was asymptomatic except for slight dizziness. Transesophageal echocardiography (ECG) and 24-hour ECG were negative for cardiac embolus. In regard to medical history, 6 weeks prior to admission the patient experienced several short episodes of vertigo and double vision while rotating her head. Computed tomography angiography (CTA) revealed irregular stenosis of the left VA at the level C5-C6 caused by a massive posterior osseous prominent spondylophyte of the facet joint C5C6 on the side of the dominant VA (Figs. 2a, b and 3a). The patient was discharged with an oral anticoagulant and instructed to avoid extreme head rotation.

Neurovascular ultrasound two months later showed persistent high-grade stenosis of the V2 segment of the VA. Because of the persistent stenosis and the cerebellar stroke on the left side due to either a V2 thrombosis as a result of arterial stasis or microdissection as well as the presence of contralateral VA hypoplasia, we opted for a surgical approach to relieve the osseous stenosis. The longus colli muscle 

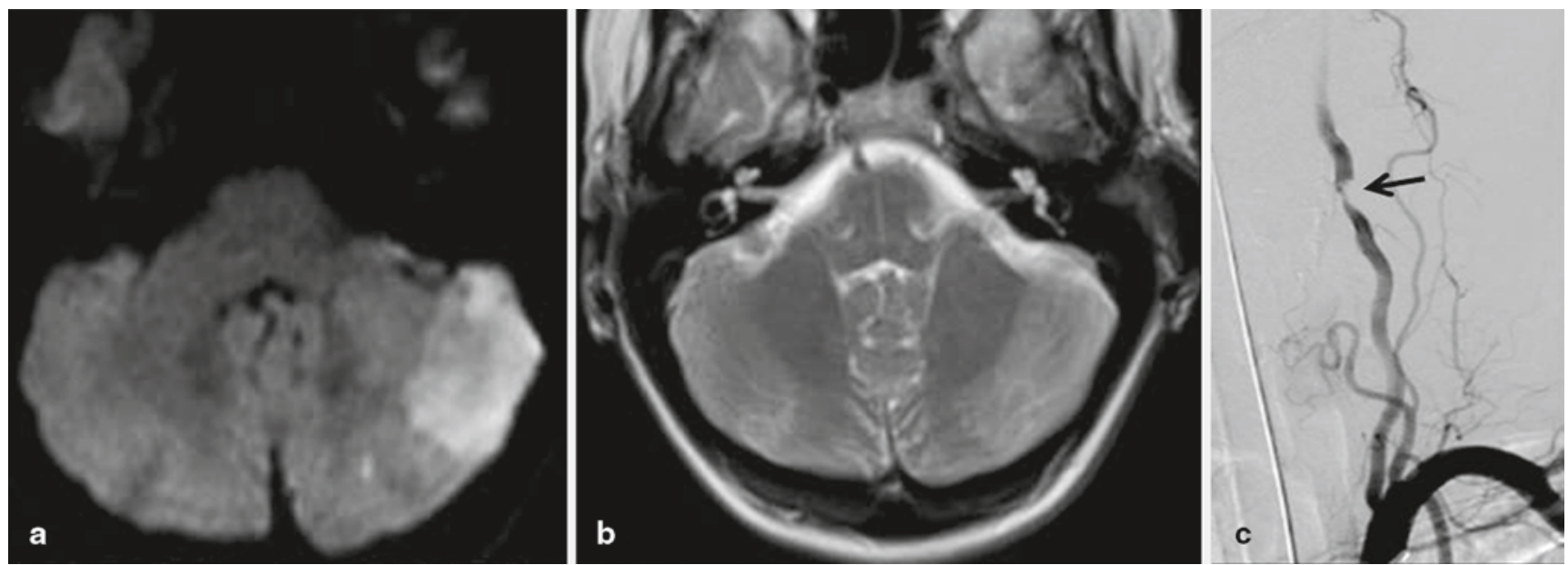

Fig. 1 a Hyperintense signal on diffusion-weighted imaging map reveals PICA territory cerebellar infarction on the left side. b T2 weighted

lesion. c DSA of the left vertebral artery demonstrates eccentric and image with no $\mathrm{T} 2$ hyperintensity corresponding to an acute ischemic

Fig. 2 a Computed tomography arteriogram (CTA) shows narrow but existing vertebral artery opacification of the left vertebral artery in the V2 segment (arrow) in the neutral position. b The 3D reconstruction of the CTA illustrates the osseous prominence at the facet joint of $\mathrm{C} 5-\mathrm{C} 6$ on the left side compressing the corresponding V2 segment (asterisk)
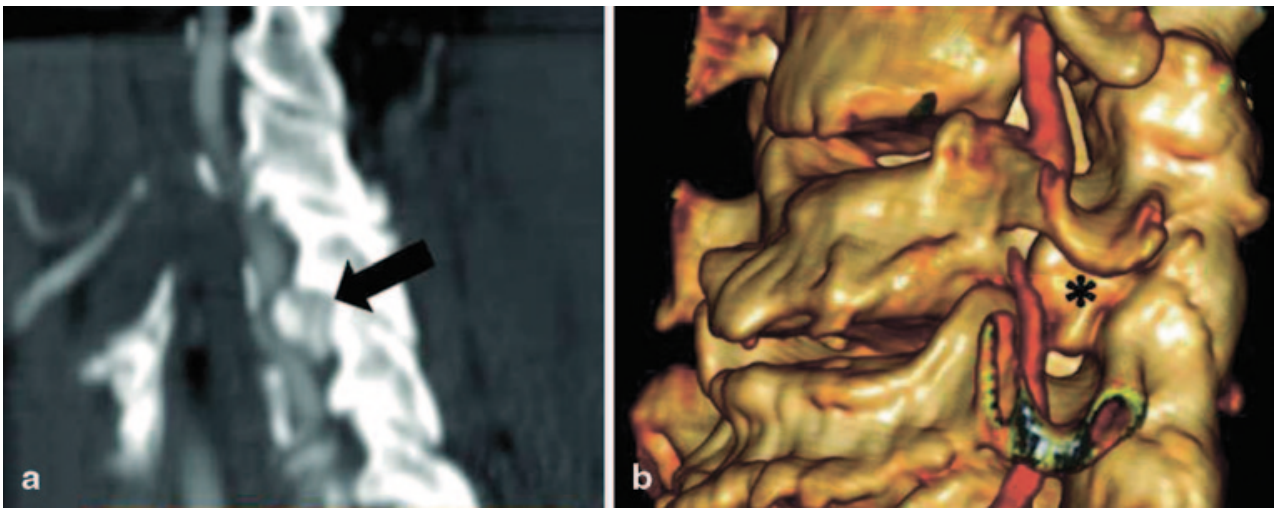

Fig. 3 a Preoperative CTA scan at the level C5-C6 shows the massive osseous prominence (arrow) at the facet joint on the left side with narrowing of the neuronal foramen and VA compression. b The postoperative scan illustrates the normalizing effect of surgical decompression on the VA on the left side
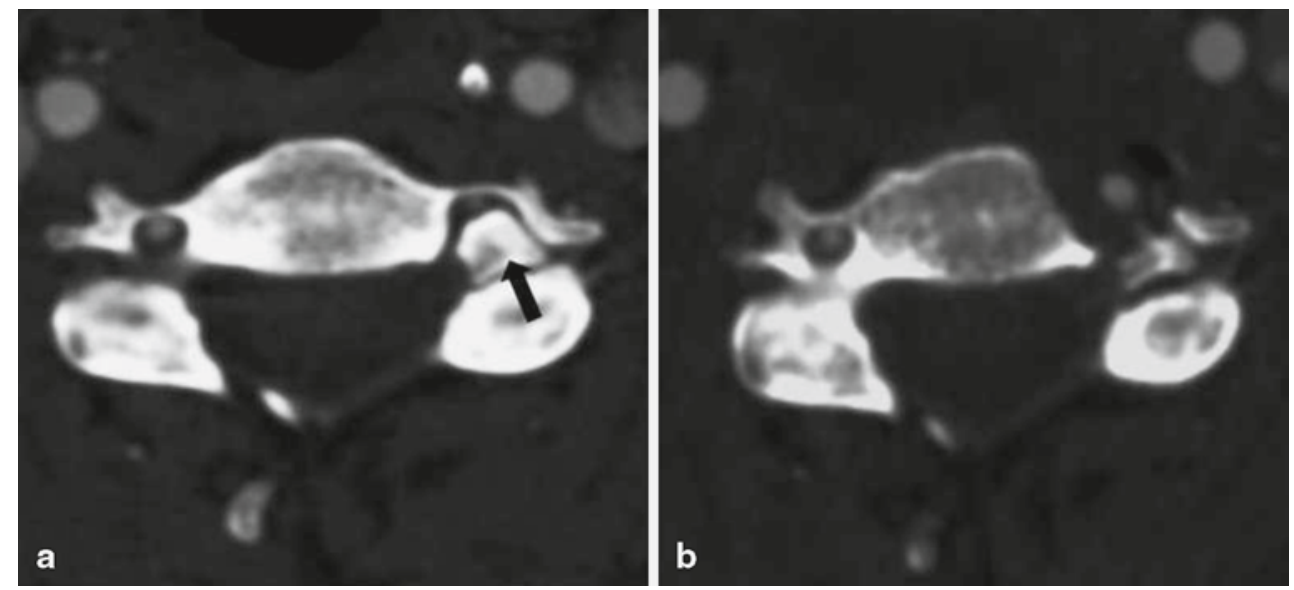

was mobilized laterally and divided at the C5-C6 level interspaces. At the $\mathrm{C} 5$ and $\mathrm{C} 6$ level, the lateral portion of the vertebral body as well as the anterior surface of the transverse foramina were removed to mobilize the V2 segment laterally and gain access for the osteophytectomy. Postoperative CTA showed a decompressed normal V2 segment of the VA on the left dominant side (Fig. 3b). The patient was discharged with residual slight ataxia and experienced no further vertebrobasilar transient ischemic attacks (TIA) throughout the six months of follow-up.

\section{Discussion}

Here we report a patient with rotational occlusion of the VA by an osseous prominence at the $\mathrm{C} 5-\mathrm{C} 6$ facet joint on the left 
side that resulted in a thrombus formation in the V2 segment of the VA, probably due to hemostasis or microdissection during neck rotation to the right side. Ipsilateral ischemic stroke in the vessel territory of the PICA occurred and was caused by either arterio-arterial embolism or by hemodynamic impairment. Ischemic stroke was preceded by recurrent vertebrobasilar TIAs provoked by head rotation. Contralateral VA hypoplasia was diagnosed as the predisposing factor for hemodynamic impairment of the posterior circulation during head rotation. Symptomatic rotational VA occlusion of the V2 segment is a rare condition. In normal circumstances, there is sufficient collateral blood supply provided by the contralateral VA as well as the anterior circulation when the circle of Willis is well developed. However, symptoms may develop in cases where contralateral VA hypoplasia, stenosis or occlusion or additional stenosis of the proximal VA are present.

Reports on treatment options for rotational VA syndrome are limited to a few case reports and small surgical or endovascular series [7-9]. Surgical decompression of the VA, endovascular stenting of the concerning artery or treatment of the contralateral atherosclerotic VA have been proposed $[8,10,11]$. There are a few published cases that conservatively treat the condition by restricting head rotation $[12,13]$. This latter approach may be limited to the fact that surgical treatment on one hand has been shown to be effective and further morbidity could be avoided. Nearly $50 \%$ of patients treated conservatively went on to have infarction or suffered residual neurological deficits [1]. An $85 \%$ success rate for surgery, based on the relief of symptoms, has been reported [1]. In our case, repeated angiography and ultrasound examination after thrombolysis revealed persistent stenosis on the affected side as well as a hypoplastic VA on the contralateral side. Due to the fact that symptomatic stroke occurred in the vessel territory of the affected side, surgical treatment was considered. Factors influencing treatment decisions may include stroke type, location of occlusion, collateral circulation, and age. Location of occlusion is especially relevant for choosing the appropriate treatment. The VA is anatomically divided into four segments V1-V4, of which the V2 segment corresponds to the intervertebral portion of the transverse foramen C3-C6. Stenosis of the V2 segment is most often due to extrinsic compression. In most cases, surgical treatment includes an anterior approach for removal of the lateral osteophytes, an uncectomy and (partial) transversectomy as well as consecutive osteophytectomy as described by Nagashima et al. [14]. In rare cases, compression of the V2 segment is due to a laterally herniated intervertebral disc $[5,9]$. In the V3 segment of the VA at the $\mathrm{C} 1-\mathrm{C} 2$ level, partial unroofing of the transverse foramina $[1,9,15]$ or cervical fusion $[9,16,17]$ or a combination of both has been proposed [7]. Less commonly, the proximal portion of the VA in the V1 segment is constricted by a fibrous band [18] Recently, there are a few case reports describing treatment of this entity with endovascular interventions. Nowadays, therapies tend to be less invasive and endovascular treatment gains further acceptance as the first-line treatment. Natello et al. [11] describes successful treatment of a patient with bow hunter's syndrome with stent placement. In two other reports of bow hunter's syndrome, stent placement in contralateral atherosclerotic VA stenosis was performed and resulted in relief of symptoms $[8,10]$. Nevertheless, treating an extrinsic compression of vessels by osteophyte formation with stents can result in severe in-stent stenosis due to the dynamic character of the compressive mechanism. Fischer et al. [19] reported a case of VA dissection due to cervical spondylosis at the V2 segment treated with a drug-eluting balloon-expandable stent with consecutive restenosis two days later. Restenosis was treated by insertion of a balloonexpandable covered stent. Follow-up angiography eight weeks later showed a persisting high-grade in-stent restenosis. Finally, a CT scan of the cervical spine depicted a massive spondylosis at C6-C7 with narrowing of the neuronal foramen and constriction of the stent by a large osteophyte at the transverse foramen. Even with increased intraluminal pressure of stents, a certain resistance to extrinsic compressive force may be sustained. However because of dynamic forces resulting from rotation of the neck, it is questionable whether there is enough resistance of the stent to render the vessel open in this dynamic process. Therefore, we believe that in the rare case of positional VA occlusion in the V2 segment, a surgical approach is preferred whenever feasible. Potentially increasing osteophytic hypertrophy and possible in-stent restenosis either by scar tissue formation [20] or extrinsic compressive restenosis [19] may occur. The latter increases the risk of hypoperfusion, stasis, thrombus and emboli formation as well as thrombotic occlusion [21, 22]. Moreover, decision-making may be difficult due to the fact that the dominant VA is mostly affected and thus iatrogenic occlusion of the treated artery may have devastating consequences.

In conclusion, treatment strategies in patients with bow hunter's syndrome must be considered on an individual basis depending on location and status of the affected vessel, collateral circulation, osseous abnormalities, comorbidities, and patient's daily life activities.

Acknowledgments The authors would like to thank Department of Neurosurgery, Publications Office, for proofreading the final manuscript.

Conflict of Interest No funding was received for this work. The authors declare no conflict of interest. 


\section{References}

1. Kuether TA, Nesbit GM, Clark WM. Rotational vertebral artery occlusion: a mechanism of vertebrobasilar insufficiency. Neurosurgery. 1997;41:427-32.

2. Morimoto T, Kaido T, Uchiyama Y, Tokunaga H, Sakaki T, Iwasaki S. Rotational obstruction of nondominant vertebral artery and ischemia. Case report. J Neurosurg. 1996;85:507-9.

3. Wakayama K, Murakami M, Suzuki M, Ono S, Shimizu N. Ischemic symptoms induced by occlusion of the unilateral vertebral artery with head rotation together with contralateral vertebral artery dissection — case report. J Neurol Sci. 2005;236:87-90.

4. Yang PJ, Latack JT, Gabrielsen TO, Knake JE, Gebarski SS, Chandler WF. Rotational vertebral artery occlusion at C1-C2. AJNR Am J Neuroradiol. 1985;6:96-100.

5. Vates GE, Wang KC, Bonovich D, Dowd C F, Lawton MT. Bow hunter stroke caused by cervical disc herniation. Case report. J Neurosurg. 2002;96:90-3.

6. Dabus G, Gerstle RJ, Parsons M, Cross DT, Moran CJ, Thompson $\mathrm{R}$, et al. Rotational vertebrobasilar insufficiency due to dynamic compression of the dominant vertebral artery by the thyroid cartilage and occlusion of the contralateral vertebral artery at C1-2 level. J Neuroimaging. 2008;18:184-7.

7. Miele VJ, France JC, Rosen CL. Subaxial positional vertebral artery occlusion corrected by decompression and fusion. Spine (Phila Pa 1976). 2008;33:E366-70.

8. S ugiu K, Agari T, Tokunaga K, Nishida A, Date I. Endovascular treatment for bow hunter's syndrome: case report. Minim Invasive Neurosurg. 2009;52:193-5.

9. Vilela MD, Goodkin R, Lundin DA, Newell DW. Rotational vertebrobasilar ischemia: hemodynamic assessment and surgical treatment. Neurosurgery. 2005;56:36-43.

10. Gomi M, Hattori I, Horikawa F, Iwasaki K. A case of bow hunter's stroke treated with endovascular surgery. No Shinkei Geka. 2006;34:189-92.
11. Natello GW, Carroll CM, Katwal AB. Rotational vertebrobasilar ischemia due to vertebral artery dynamic stenoses complicated by an ostial atherosclerotic stenosis. Vasc Med. 2009;14:265-9.

12. Hayashi K, Matsuo T, Kurihara M, Shibata S. A case of juvenile bow hunter's stroke. No To Shinkei. 2000;52:431-4.

13. Owolabi MO, Ogah OS, Ogunniyi A. Episodic vertigo resulting from vascular risk factors, cervical spondylosis and head rotation: two case reports. Neuropsychiatr Dis Treat. 2007;3:675-8.

14. Nagashima C. Surgical treatment of vertebral artery insufficiency caused by cervical spondylosis. J Neurosurg. 1970;32:512-21.

15. Shimizu T, Waga S, Kojima T, Niwa S. Decompression of the vertebral artery for bow-hunter's stroke. Case report. J Neurosurg. 1988;69:127-31.

16. Matsuyama T, Morimoto T, Sakaki T. Usefulness of three-dimensional CT for bow hunter stroke. Acta Neurochir (Wien). 1997;139:265-6.

17. Matsuyama T, Morimoto T, Sakaki T. Comparison of C1-2 posterior fusion and decompression of the vertebral artery in the treatment of bow hunter's stroke. J Neurosurg. 1997;86:619-23.

18. Mapstone T, Spetzler RF. Vertebrobasilar insufficiency secondary to vertebral artery occlusion from a fibrous band. Case report. J Neurosurg. 1982;56:581-3.

19. Fischer S, Schul C, Lanzer P, Henkes H. Cervical vertebral artery dissection and recurrent in-stent stenosis due to $\mathrm{C} 6 / 7$ foraminal spondylosis. Clin Neuroradiol. 2010;20:117-21.

20. Mehran R, Dangas G, Abizaid AS, Mintz GS, Lansky AJ, Satler LF, et al. Angiographic patterns of in-stent restenosis: classification and implications for long-term outcome. Circulation. 1999;100:1872-8.

21. Sullivan HG, Harbison JW, Vines FS, Becker D. Embolic posterior cerebral artery occlusion secondary to spondylitic vertebral artery compression. Case report. J Neurosurg. 1975;43:618-22.

22. Berguer R, Morasch MD, Kline RA. A review of 100 consecutive reconstructions of the distal vertebral artery for embolic and hemodynamic disease. J Vasc Surg. 1998;27:852-9. 